\title{
3D Analysis of Radiofrequency-Ablated Tumors in Liver: A Computer-Aided Diagnosis Tool for Early Detection of Local Recurrences
}

\author{
Ivan Bricault ${ }^{1,2}$, Ron Kikinis ${ }^{1}$, Eric vanSonnenberg ${ }^{1}$, \\ Kemal Tuncali ${ }^{1}$, and Stuart G. Silverman ${ }^{1}$ \\ ${ }^{1}$ Surgical Planning Lab / Department of Radiology, Brigham and Women's Hospital, 75 \\ Francis Street, Boston, MA 02115, USA \\ 2 TIMC-IMAG laboratory, University Hospital, Grenoble, France \\ Ivan.Bricaultaimag.fr
}

\begin{abstract}
Radiofrequency ablation is being used increasingly in the treatment of liver tumors, and the detection of local recurrences on follow-up imaging is an important and occasionally challenging task. When the tumor is not associated with nodular enhancement, recurrence detection only relies on a precise identification of shape changes in the post-ablation area. In order to better characterize subtle shape changes, we present a computer-aided diagnosis tool based on a semi-automatic segmentation of CT data with a watershed algorithm. The $3 \mathrm{D}$ moment of inertia of the segmented area is computed and provides a quantitative criterion for the study of post-ablation changes over time. Preliminary results on two clinical cases demonstrate that our tool can effectively improve the radiologist's ability to detect early tumor recurrence.
\end{abstract}

Radiofrequency (RF) ablation is a minimally invasive alternative to surgical resection of liver tumors. It delivers high-frequency alternating current in situ, inducing tumor thermal necrosis. In the follow-up of RF ablation, the most widely used imaging modality is contrast-enhanced computed tomography (CT). Successfully ablated tumors appear as low-attenuation areas with no enhancement; hence non-enhancing recurrences may be recognizable only by detecting subtle changes in the post-RF ablated area shape [1]. This detection on follow-up CT 2D slices constitutes a challenging task for the radiologist. Since it can have important consequences for patients, it motivated the development of our computer-aided diagnosis tool.

A method for the evaluation of RF ablations has been previously presented [2]. Whereas this study used an ellipsoid model for segmentation and was focused on the immediate post-ablation comparison between MRI and histological data, we address here the different clinical problem of long-term follow-up CT interpretation. Moreover, since we want to detect shape changes, our segmentation method is not restricted to a particular geometric model.

Our software is based on the National Library of Medicine's Insight toolkit (www. itk.org). A curvature flow filter is used for edge-preserving CT smoothing. A watershed algorithm, controlled by a user interactively defined segmentation level, is able to segment the post-RF ablated area. The resulting 3D object is post-processed by mathematical morphology erosion and dilatation filters. Because a normal postablation area usually shrinks and becomes smoother over time, the moment of inertia 
has been chosen to characterize its shape in addition to $3 \mathrm{D}$ volume. The total procedure is fast (at most a few minutes) and requires minimal user interaction.

We retrospectively analyzed the case of a 47 year-old female presenting multiple liver metastases from a gastro-intestinal stromal tumor (patient \#1). Follow-up axial CT scans were acquired at months $\mathrm{M}=1,5,6,9,12$ and 15 after the RF ablation of one active metastasis. Whereas the radiologist detected an increase in size of the postablated area only at $\mathrm{M}=12$, our computer tool demonstrated visually and quantitatively (Fig. 1) a definitive suggestion of recurrence at $\mathrm{M}=9$. Tumor recurrence was confirmed by a PET scan at that time. In the case of patient \#2, no recurrence was observed. The evolution chart in Fig. 1 confirms the success of this ablation. In both patients, Fig. 1 chart additionally shows that 3D moment of inertia can provide a more sensitive criterion than 3D volume, since inertia presents larger scale variations more dependent on peripheral shape deformations.

In conclusion, these preliminary results demonstrate the clinical effectiveness of 3D analysis for early recurrence detection. It is currently being further validated by studying the evolution patterns of post-RF areas in a larger series of patients.
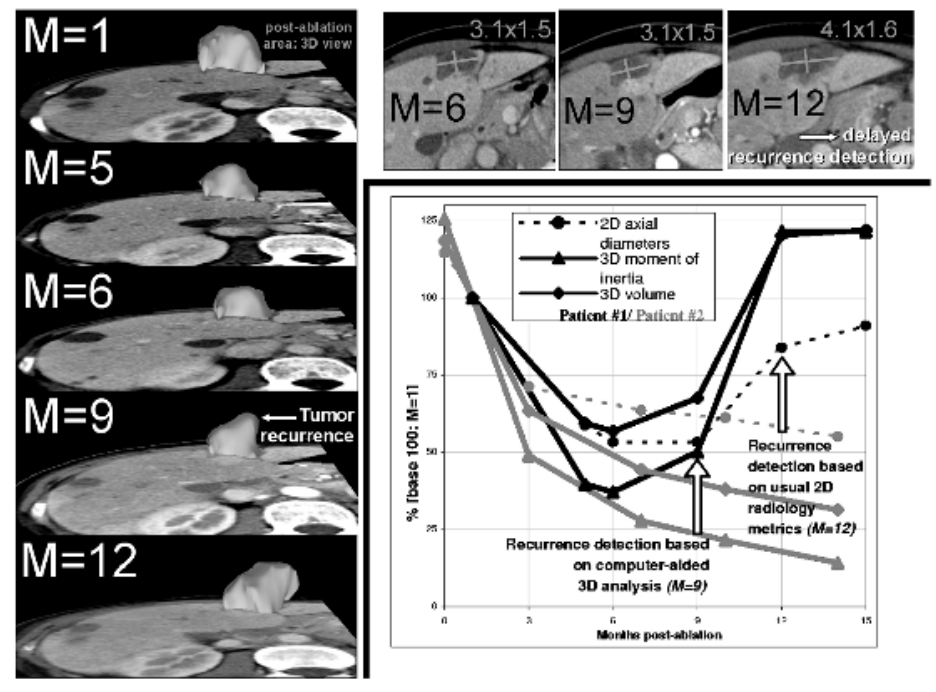

Fig. 1. Ablated area, patient \#1: evolution of 3D segmented shape (left) and axial diameters (top right) over time ( $M=$ months after radiofrequency treatment).

Bottom right: Comparison of recurrence detection criteria, patients \#1 and \#2.

\section{References}

1. Choi, H., Loyer, E.M., DuBrow, R.A., Kaur, H., David, C.L., Huang, S., Curley, S., Charnsangavej, C.: Radio-frequency ablation of liver tumors: assessment of therapeutic response and complications. Radiographics 21 (2001) S41-S54

2. Lazebnik, R.S., Breen, M.S., Lewin, J.S., Wilson, D.L.: Automatic Model-Based Evaluation of Magnetic Resonance-Guided Radio Frequency Ablation Lesions with Histological Correlation. J. Magn. Reson. Imaging. 19 (2004) 245-354

This project was supported in part by a study grant from the French Radiological Society. 